\title{
Respuesta en parámetros de intercambio gaseoso y supervivencia en plantas jóvenes de genotipos comerciales de Eucalyptus spp sometidas a déficit hídrico
}

\author{
Gas-exchange response and survival of young Eucalyptus spp commercial \\ genotypes under water stress
}

\author{
María Silva a $^{\text {, Rafael Rubilar a,b*, Juan Espinoza }}{ }^{\text {, }}$, Marco Yánez ${ }^{\text {, }}$, Verónica Emhart c, Juan José Quiroga ${ }^{\text {d }}$ \\ *Autor de correspondencia: ${ }^{a}$ Universidad de Concepción, Facultad de Ciencias Forestales, Departamento de Silvicultura, \\ Victoria Nº631, Concepción, Chile, tel.: 56-41-2204980, rafaelrubilar@udec.cl

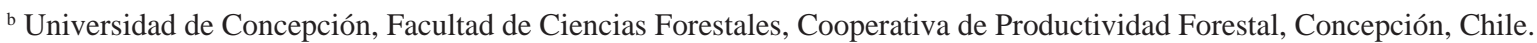 \\ c Forestal Mininco S.A., Avenida Alemania 751, Los Ángeles, Chile. \\ ${ }^{\mathrm{d}}$ Bioforest S.A., Camino a Coronel km 15 s/n, Coronel, Chile.
}

\begin{abstract}
SUMMARY
In the last decades, breeding programs for Eucalyptus species in Chile have mainly focused on increasing productivity and adaptation. However, little is known about how droughts and climate change may influence physiological responses that determine tree growth. Evaluating leaf-level physiology may be important to predict survival of eucalyptus genotypes under severe water stress. This study assessed net photosynthesis $\left(A_{N}\right)$, stomatal conductance $\left(g_{s}\right)$, transpiration $(E)$ and instantaneous water use efficiency (WUE $)$ in Eucalyptus globulus, E. nitens, E. badjensis, E. smithii and E. globulus x E. nitens and E. camaldulensis x E. globulus hybrids genotypes under increasing water stress conditions. Survival of each genotype was related to the observed physiological responses at -0.03 MPa, -1.5 MPa and -2.5 MPa soil water potentials. Higher plant survival was observed on genotypes with reduced water loss due to stomatal adjustment under water deficit, and on genotypes that could maintain initial photosynthetic rates under increased water deficit despite reductions in stomatal conductance. A negative relationship between plant survival and gas-exchange parameters suggests that photosynthesis rate, under high water availability (-0.03 MPa) and moderate water deficit (-1.5 MPa), may be a useful indicator for early selection of genotypes at sites under water limitations.
\end{abstract}

Key words: water stress, carbon assimilation, water use, clones, hybrids.

\section{RESUMEN}

En los últimos años, la mejora genética de especies de Eucalyptus en Chile se ha enfocado en el aumento de la productividad y mejor adaptación a condiciones de sitio limitantes para el crecimiento. Sin embargo, se desconocen las respuestas fisiológicas que condicionan este comportamiento frente a eventos de sequía. Estimaciones fisiológicas a nivel de planta pueden ser de gran relevancia para predecir la supervivencia de genotipos de Eucalyptus ante eventos de estrés hídrico severo. En el presente estudio se evaluó la fotosíntesis neta $\left(A_{N}\right)$, transpiración $(E)$, conductancia estomática $\left(g_{s}\right)$ y eficiencia instantánea en el uso de agua $\left(E U A_{i}\right)$ en genotipos de Eucalyptus globulus, E. nitens, E. badjensis, E. smithii e híbridos E. globulus x E. nitens y E. camaldulensis x E. globulus en potenciales hídricos del substrato de - $0,03 \mathrm{MPa},-1,5 \mathrm{MPa}$ y $-2,5 \mathrm{MPa}$. La supervivencia de cada material genético se relacionó con las variables fisiológicas mencionadas. Los resultados obtenidos muestran que los genotipos con mayor supervivencia son aquellos que, en condiciones de déficit hídrico, controlan la pérdida de agua a través de ajuste estomático; además de los genotipos capaces de mantener altas tasas fotosintéticas al aumentar el déficit hídrico. La relación negativa observada entre la supervivencia y las variables de intercambio gaseoso, en particular la fotosíntesis, sugiere que la evaluación de estos parámetros en condiciones de alta disponibilidad de agua (-0,03 $\mathrm{MPa})$ y estrés hídrico moderado $(-1,5 \mathrm{MPa})$ puede ser de utilidad para la selección de genotipos con mayor éxito al establecimiento en sitios con limitantes hídricas.

Palabras clave: estrés hídrico, asimilación de carbono, uso de agua, clon, híbrido.

\section{INTRODUCCIÓN}

Las plantaciones del género Eucalyptus se han expandido mundialmente logrando cubrir una superficie aproximada de 20 millones de hectáreas (Hubbard et al.
2010). En Chile, las plantaciones de este género son de gran importancia económica alcanzando las 800 mil hectáreas plantadas al año 2013 (INFOR 2015). Las principales especies plantadas son Eucalyptus globulus Labill y E. nitens $\mathrm{H}$. Deane et Maiden, siendo E. globulus seleccionado 
por su mejor rendimiento pulpable, mientras que E. nitens por su alta tasa de crecimiento en ambientes más fríos. Durante los últimos años se ha desarrollado un creciente interés por la selección de genotipos de mayor productividad y mejor calidad de madera, con la incorporación de la mejora genética enfocada al desarrollo de híbridos entre estas y otras especies. Sin embargo, no se ha tenido en consideración la evaluación de las características fisiológicas y genéticas que establecen las diferencias en el rendimiento de los distintos genotipos de interés productivo, y que regulan su crecimiento y productividad en distintas condiciones climáticas y edáficas (Navarrete et al. 2013).

En la actualidad, el déficit hídrico, acrecentado por las posibles consecuencias del cambio climático, es considerado el principal factor ambiental limitante del crecimiento y productividad de plantaciones con fines comerciales, especialmente en climas mediterráneos o en ambientes semiáridos (Chaves et al. 2003) Por ello existe preocupación acerca del efecto que este pueda causar sobre la productividad de las plantaciones en distintos ambientes de crecimiento (Chaves et al. 2009). Por otra parte, la necesidad de aumentar la superficie plantada en Chile, ha generado que el establecimiento de plantaciones en Chile se realice en sitios cada vez más restrictivos para el crecimiento, como lo son el secano costero y la precordillera de Los Andes (Coopman et al. 2008).

Las respuestas de las plantas a la escasez de agua son complejas, incluyendo cambios adaptativos y/o efectos deletéreos genotipo (Chaves et al. 2002). Las estrategias para hacer frente al estrés hídrico generalmente implican una serie de mecanismos cruzados entre estrategias para evitar y/o tolerar el estrés, las cuales interactúan sinérgica y antagónicamente con otros estreses, además de variar con el genotipo (Chaves et al. 2002). Estas respuestas pueden ser a nivel fisiológico y morfológico tales como cambios en la estructura de la planta, tasas de crecimiento, eficiencia en el uso de agua, potencial tisular osmótico, la conductancia estomática (Li y Wang 2003), reducción del potencial hídrico de prealba, y aumento en la concentración de osmolitos, antioxidantes y pigmentos del ciclo de las xantófilas en hojas y tallos (Navarrete et al. 2013).

Estos mecanismos tienden a restringir la productividad potencial de plantaciones por inhibición de la expansión celular (Costa e Silva 2004) y, principalmente, por la disminución del balance de carbono en la planta el cual es altamente dependiente de la fotosíntesis (Flexas et al. 2009). Por ello, las respuestas fotosintéticas en condiciones de estrés hídrico han sido ampliamente estudiadas, teniendo especial énfasis en los factores, tanto difusivos como bioquímicos, que la limitan (Chaves et al. 2002, Chaves et al. 2003, Galmés et al. 2007, Flexas et al. 2009). Bajo condiciones de estrés hídrico moderado, la conductancia estomática ha sido reportada ser la principal causa de disminución de fotosíntesis (Medrano et al. 2002, Chaves et al. 2003). Sin embargo, la disminución de la tasa fotosintética no solo es explicada por este factor, sino que también por otros como cambios en la de difusión del $\mathrm{CO}_{2}$ y en la conductancia de este a través del mesófilo (Flexas et al. 2009). Algunas investigaciones señalan que la buena correlación existente entre conductancia estomática y fotosíntesis permite usarla como referencia para evaluar estrés hídrico (Medrano et al. 2002, Pita et al. 2005, Eksteen et al. 2013) y, en consecuencia, evaluar los efectos de la mejora genética en la resistencia a la sequía sobre el crecimiento (Wikberg y Ögren 2004). El análisis de estos procesos fisiológicos que regulan los flujos de agua en las plantas (absorción y pérdida) permiten cuantificar la eficiencia con la que estas usan el agua. De este modo se define la eficiencia en el uso de agua (EUA), como el volumen de agua que las plantas necesitan consumir (evapotranspiración) para incorporar a su biomasa una determinada cantidad de carbono proveniente de la atmósfera (Medrano et al. 2007). Las medidas de intercambio de gases en hojas permiten determinar la fotosíntesis neta y la transpiración, y con ello la eficiencia instantánea en el uso del agua $\left(E U A_{i}\right)$ a escala foliar como el cociente de estos dos parámetros (Medrano et al. 2007). En estudios de la variación de la $E U A_{i}$ en distintos genotipos y condiciones de disponibilidad de agua en el suelo se ha reportado que las diferencias encontradas dependen de la estrategia de respuesta de los genotipos al déficit hídrico, atribuidas a variaciones en el intercambio gaseoso (Pita et al. 2005, Medrano et al. 2007, Navarrete et al. 2013) y ha sido considerado de interés para evaluar la adaptación de genotipos a condiciones de sequía ( Li 2000, Dye 2000).

Los parámetros de intercambio gaseoso (fotosíntesis, conductancia estomática y transpiración) y su efecto sobre la eficiencia instantánea del uso de agua se han identificado como herramientas potenciales para seleccionar genotipos con mejor comportamiento frente al estrés hídrico, pero existe discusión acerca de cuáles son los más apropiados dado que el crecimiento y uso de agua dependen también de la especie, edad e interacciones con el medio ambiente (Pardos 2007). La identificación de los parámetros, que en etapas tempranas de desarrollo, permitan mejorar los criterios de selección de genotipos productivos, resistentes a la sequía y más eficientes en el uso de agua, son relevantes dado las condiciones climáticas cambiantes que restringen el crecimiento y sobrevivencia de las plantas en el establecimiento. Para ello, es necesario generar información que permita comprender la relación entre la respuesta fisiológica de nuevos genotipos seleccionados en etapas tempranas de desarrollo, las condiciones del sitio para su establecimiento y la posibilidad de evaluar el riesgo de supervivencia y desarrollo temprano de cada material frente a condiciones de estrés hídrico.

La hipótesis propuesta en el presente estudio establece que las variables de intercambio gaseoso: conductancia estomática, fotosíntesis instantánea y transpiración, en plántulas sometidas a distintos niveles de estrés hídrico, permiten identificar genotipos de Eucalyptus spp. que presentarán mayor supervivencia en sitios con disponibilidad 
hídrica limitante en etapas tempranas de desarrollo. Para ello se evalúa la respuesta en intercambio gaseoso y su efecto en la eficiencia instantánea en el uso de agua para 35 genotipos comerciales de E. globulus, E. nitens, E. badjensis Beuzev et M.B. Welch, E. smithii R.T. Baker e híbridos E. globulus $x$ E. nitens y E. camaldulensis Dehnh x E. globulus sometidos a condiciones crecientes de estrés hídrico y la relación de cada parámetro de intercambio gaseoso evaluado con la supervivencia de cada material genético.

\section{MÉTODOS}

Material vegetal. El estudio consideró 35 genotipos de Eucalyptus globulus (Eg1-Eg24), E. nitens (En1, En2), E. badjensis (Eb), E. smithii (Es) e híbridos E. globulus $x$ E. nitens (Egn1-Egn6) y E. camaldulensis x E. globulus (Ecg) desarrollados por Forestal Mininco S.A. y Arauco S.A con fines comerciales. Las plantas se produjeron en contenedores de $110 \mathrm{~cm}^{3}$ con sustrato de corteza de pino (Pinus radiata D. Don) compostada según los criterios establecidos por las empresas. Después de cuatro meses de desarrollo, 36 plantas por genotipo se seleccionaron para el ensayo. Para la selección de plantas se consideraron características morfológicas (altura y diámetro de cuello) y ausencia de daños o enfermedades. Estas plantas se establecieron en cajas de poliestireno expandido de $0,033 \mathrm{~m}^{3}$ con sustrato de compost de corteza de pino.

La aclimatación al medio de crecimiento tuvo una duración de tres semanas previas al inicio del ensayo. Para ello, las plantas se regaron cada dos días, manteniendo el sustrato entre un 75-100 \% del contenido de humedad equivalente a capacidad de campo (-0,03 MPa). Cada siete días se realizó fertilización a nivel de sustrato con Phostrogen ${ }^{\circledR}$ (NPK 13-10-27, S, Mg, Ca, Fe, B, Mn, Cu y Mo, Bayer Garden, Cambridge, UK) a una concentración de $1 \mathrm{~g}$ por cada litro de agua. Posterior a este período se establecieron dos tratamientos diferenciados por gradiente de humedad.

El ensayo se llevó a cabo en el vivero experimental de la Universidad de Concepción entre el 13 de enero y el 7 de febrero de 2014, periodo de tiempo necesario para que las plantas alcanzaran las condiciones de estrés hídrico establecidas para la realización de las mediciones y muestreos correspondientes (ver detalle más adelante).

Establecimiento de tratamientos. Se establecieron dos tratamientos con gradientes de humedad contrastantes: tratamiento control, consistente en mantener el riego en condiciones de capacidad de campo (-0,03 MPa); y sequía, donde se eliminó por completo el riego para obtener la disminución progresiva de la cantidad de agua disponible para las plantas en el medio de crecimiento. En ambos tratamientos las plantas se cubrieron para impedir la adición no controlada de agua por precipitación. Dos cajas por genotipo, cada una con 12 plantas, se establecieron para cada tratamiento. El monitoreo del contenido de humedad del sustrato se realizó cada dos días con un equipo TDR (Time Domain Reflectometry) previamente calibrado en base al contenido de humedad gravimétrico $\left(\mathrm{CH}_{\mathrm{w},} \mathrm{g}\right.$ de agua / g de sustrato) de muestras de sustrato a distintos niveles de humedad, los cuales fueron posteriormente asociados con la curva de retención de humedad del sustrato que estableció los potenciales hídricos del sustrato $\left(\psi_{\text {sustrato }}\right)$ críticos para el experimento.

Instancias de muestreo. A partir de la disminución del contenido de humedad del tratamiento de sequía, se determinaron tres instancias de muestreo en las cuales se evaluó el comportamiento fisiológico de cada material genético considerado en el experimento. Cada instancia de muestreo se estableció como el contenido de humedad del sustrato, registrado en el tratamiento de sequía, equivalente a valores de potencial hídrico del sustrato de $-0,03 \mathrm{MPa}(\mathrm{M} 0),-1,5 \mathrm{MPa}$ (M1.5) y -2,5 MPa (M2.5).

Potencial hídrico foliar de prealba. El potencial hídrico foliar de prealba $\left(\psi_{\text {prealba }}\right)$ se midió con una bomba de presión Scholander (Soil Moisture Equipment Corp, CA, USA) en horarios de pre-alba (4:00 a 6:00 h). La medición se realizó a tres plantas por tratamiento seleccionando hojas del tercio superior de las mismas. Dado que todas las especies presentaban hojas sésiles, al extraer la hoja se cortó la base de la vena principal de forma de separarla del resto de la hoja para utilizarla como peciolo.

Variables instantáneas de intercambio gaseoso. La medición de variables instantáneas de intercambio gaseoso correspondientes a conductancia estomática $\left(g_{s}\right)$, transpiración instantánea $(E)$ y tasa neta de fotosíntesis $\left(A_{N}\right)$ se realizó entre 9 y 13 h en una hoja totalmente expandida, seleccionada del tercio superior de tres plantas de cada genotipo para ambos tratamientos, utilizando un sistema analizador de intercambio gaseoso (Li-6400X Li-Cor Inc., Lincoln, NE, USA) equipado con una fuente de luz led. Durante las mediciones, se mantuvieron constantes los parámetros de saturación lumínica $(1.500 \mu \mathrm{mol}$ fotones $\left.\mathrm{m}^{-2} \mathrm{~s}^{-1}\right)$, temperatura $\left(25^{\circ} \mathrm{C}\right)$, flujo de aire $\left.(500 \mu \mathrm{mol} \mathrm{s})^{-1}\right)$ y concentración de $\mathrm{CO}_{2}\left(400 \mu \mathrm{mol} \mathrm{mol}{ }^{-1} \mathrm{CO}_{2}\right)$. Cada medición tuvo un tiempo de duración entre 3 y 5 minutos, dependiendo de la estabilidad de las variables evaluadas. La eficiencia instantánea en el uso de agua $\left(E U A_{i}\right)$ se calculó como el cociente entre la tasa neta de fotosíntesis y la transpiración instantánea $\left(A_{N} / E\right)$.

Porcentaje de supervivencia. El porcentaje de supervivencia se cuantificó dos semanas después de alcanzar potencial hídrico del sustrato crítico de -2,5 MPa. Se consideró como planta viva aquella que presentara al menos el par de hojas apicales visiblemente turgentes.

Análisis estadístico. El experimento se analizó como un diseño completamente aleatorizado con tres réplicas con- 
siderando la instancia de muestreo y el genotipo como factores. Los análisis de varianza (ANDEVA) para los parámetros de intercambio gaseoso y potencial hídrico foliar se realizaron con el procedimiento Proc GLM del software SAS versión 9.0 (SAS Institute 2002). La normalidad de los residuos se probó a través de histogramas y gráficos Q-Q normal, y la homogeneidad de varianza se evaluó a través del test de Levene. Dado que los resultados de supervivencia se estimaron en forma porcentual se usaron transformaciones de raíz cuadrada del arco-seno para su análisis. La separación de medias para las variables de intercambio gaseoso y potencial hídrico foliar se realizó mediante la prueba de Tukey $(P<0,05)$. Análisis de correlación entre las variables estudiadas se realizaron mediante el coeficiente de correlación de Pearson $(\mathrm{N}=35)$.

\section{RESULTADOS}

Potencial hídrico foliar en prealba. La respuesta en el potencial hídrico foliar para los genotipos en el tratamiento control no presentó diferencias significativas en las distintas instancias de muestreo $(P=0,9701)$, observándose potenciales cercanos a $0 \mathrm{MPa}$ durante todo el estudio (figura 1A). El tratamiento de sequía, acorde a lo esperado, registró una disminución progresiva del potencial de prealba debido al aumento del déficit hídrico del sustrato y estableciendo diferencias significativas entre las medias alcanzadas $(P<0,05)$ para las distintas instancias de muestreo (figura 1). El valor medio observado en cada instancia de muestreo fue de $-0,19 \pm 0,01 \mathrm{MPa},-0,59 \pm 0,02 \mathrm{MPa}$ y de $-2,10$ $\pm 0,06 \mathrm{MPa}$, para los muestreo en $-0,03 \mathrm{MPa},-1,5 \mathrm{MPa}$ y -2,5 MPa de potencial hídrico del sustrato, respectivamente. Entre las primeras instancias de muestreo $(-0,03$ MPa y -1,5 MPa de potencial hídrico del sustrato) solo seis genotipos (Eng6, Eg2, Eg3, Eg4, Eg15 y Eg22) disminuyeron significativamente su potencial de prealba $(P<0,05)$. En etapas de menor disponibilidad hídrica del sustrato (-1,5 MPa a -2,5 MPa), se observó una caída general del potencial hídrico foliar desde la segunda instancia de muestreo para todos los genotipos $(P<0,05)$. La magnitud de esta caída fue variable entre genotipos, por lo que en la instancia de muestreo a -2,5 MPa se observaron cinco grupos de genotipos que alcanzaron distintos potenciales medios de prealba $(P<0,05)$ correspondientes a $-1,48 \pm$ 0,02 (Eg6, Eg7, Eg10, Eg11, Eg17, Eg18, Eg20, Eg23, Eng5); -1,84 \pm 0,03 (Eg14, Eg21, Eng1, Eng2); -2,24 $\pm 0,03$ (Eg1, Eg5, Eg8, Eg9, Eg12, Eg16, Eg19, Eg24, En1, En2, Eb, Ecg); -2,78 \pm 0,03 (Eg2, Eg4, Eg22, Eng4, Eng6) y -3,07 \pm 0,06 MPa (Eg3, Eg13, Eg15, Es, Eng3).

Conductancia estomática y transpiración instantánea. Se observó una correlación positiva $(\mathrm{r}=0,89, P<0,05)$ entre la conductancia estomática y la transpiración instantánea (figura 2). Una disminución generalizada de la conductancia estomática se produjo una vez iniciado el estrés hídrico, obteniéndose valores bajo 0,12 moles de $\mathrm{H}_{2} \mathrm{O} \mathrm{m}^{-2} \mathrm{~s}^{-1}$ en las instancias de muestreo finales (figura 2A). Si bien la mayoría de los genotipos disminuyó su conductancia estomática entre cada instancia de muestreo $(P<0,05)$, cinco genotipos (Eb, En1, Eg10, Eg14 y Eg24) no presentaron esta respuesta y mantuvieron valores menores a 0,21 moles de $\mathrm{H}_{2} \mathrm{O} \mathrm{m}^{-2} \mathrm{~s}^{-1}$ a lo largo del ensayo. La respuesta en la trans-
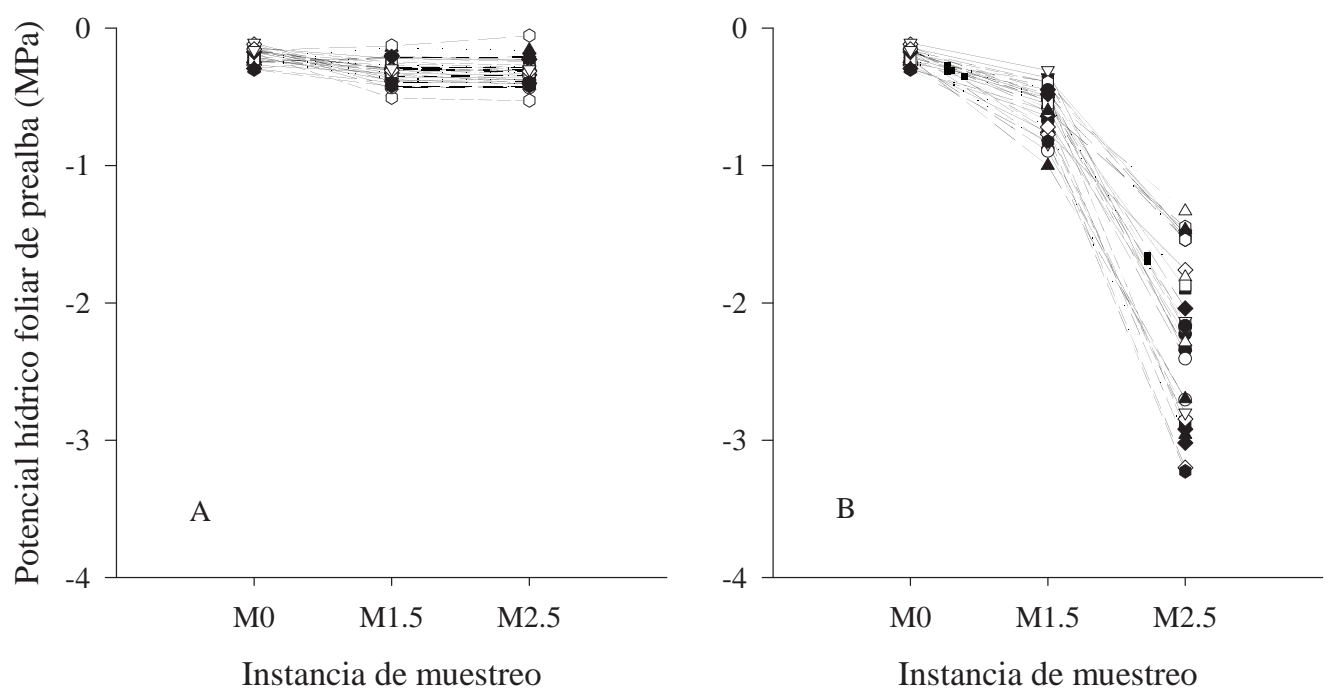

Figura 1. Media del potencial hídrico foliar de prealba medido a los 35 genotipos de Eucalyptus spp. en los tratamientos control (A) y sequía (B) en las instancias de muestreo equivalentes a -0,03 MPa (M0), -1,5 MPa (M1.5) y -2,5 MPa (M2.5) de potencial hídrico del sustrato del tratamiento de sequía.

Mean predawn water potential evaluated at -0.03 MPa (M0), -1.5 MPa (M1.5) and -2.5 MPa (M2.5) substrate water potentials for all Eucalytpus spp. $(\mathrm{n}=35)$ genotypes under control $(\mathrm{A})$ and drought $(\mathrm{B})$ treatments. 
piración instantánea fue similar (figura 2B), observándose que los genotipos Eb, En1, Eg6, Eg10 y Eg14 mantuvieron medias similares entre instancias de muestreo, sin superar los 4,3 $\mu$ moles de $\mathrm{H}_{2} \mathrm{O} \mathrm{m}^{-2} \mathrm{~s}^{-1}$ durante el ensayo.

Tasa instantánea de fotosíntesis. Se observó una correlación positiva entre la fotosíntesis instantánea y la conduc- tancia estomática $(P<0,05)$ en cada instancia de muestreo (figura 3), además de diferencias significativas $(P<0,05)$ en las tasas fotosintéticas de los genotipos en cada instancia de muestreo. Si bien la mayoría de los genotipos no presentó diferencias significativas en sus tasas de fotosíntesis entre instancias de muestreo (figura 4A), dos grupos presentaron disminuciones significativas a lo largo del
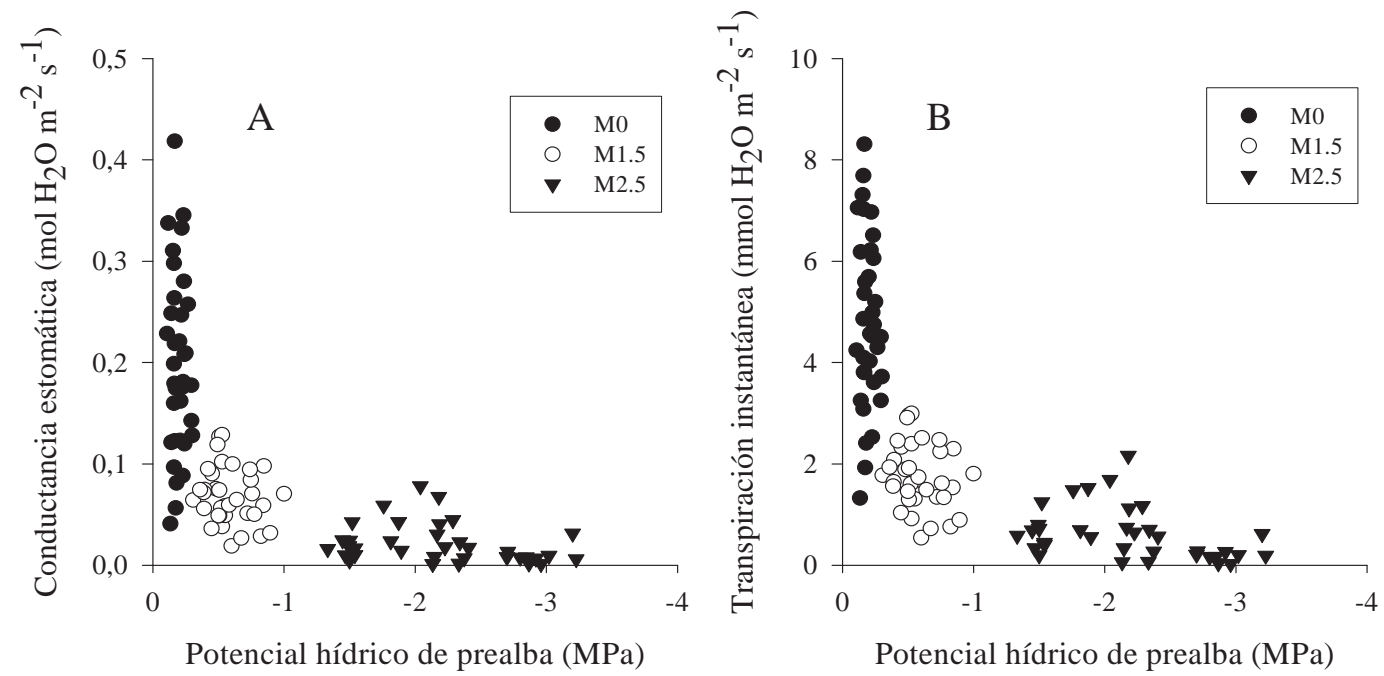

Figura 2. Variación de la media de la conductancia estomática (mol $\left.\mathrm{H}_{2} \mathrm{O} \mathrm{m}^{-2} \mathrm{~s}^{-1}\right)($ A) y de la media de la transpiración instantánea $\left(\mu \mathrm{mol} \mathrm{H} \mathrm{O} \mathrm{m}^{-2} \mathrm{~s}^{-1}\right)(\mathrm{B})$ con respecto a la media del potencial hídrico foliar de prealba (MPa) observado en los 35 genotipos de Eucalyptus spp. medidos en el tratamiento de sequía en las instancias de muestreo equivalentes a -0,03 MPa (M0), -1,5 MPa (M1.5) y -2,5 MPa (M2.5) de potencial hídrico del sustrato.

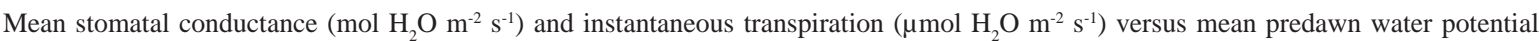
$(\mathrm{MPa})$ evaluated for all Eucalytpus spp. $(\mathrm{n}=35)$ genotypes at $-0.03 \mathrm{MPa}(\mathrm{M} 0),-1.5 \mathrm{MPa}(\mathrm{M} 1.5)$ and $-2.5 \mathrm{MPa}(\mathrm{M} 2.5)$ substrate water potential treatments.
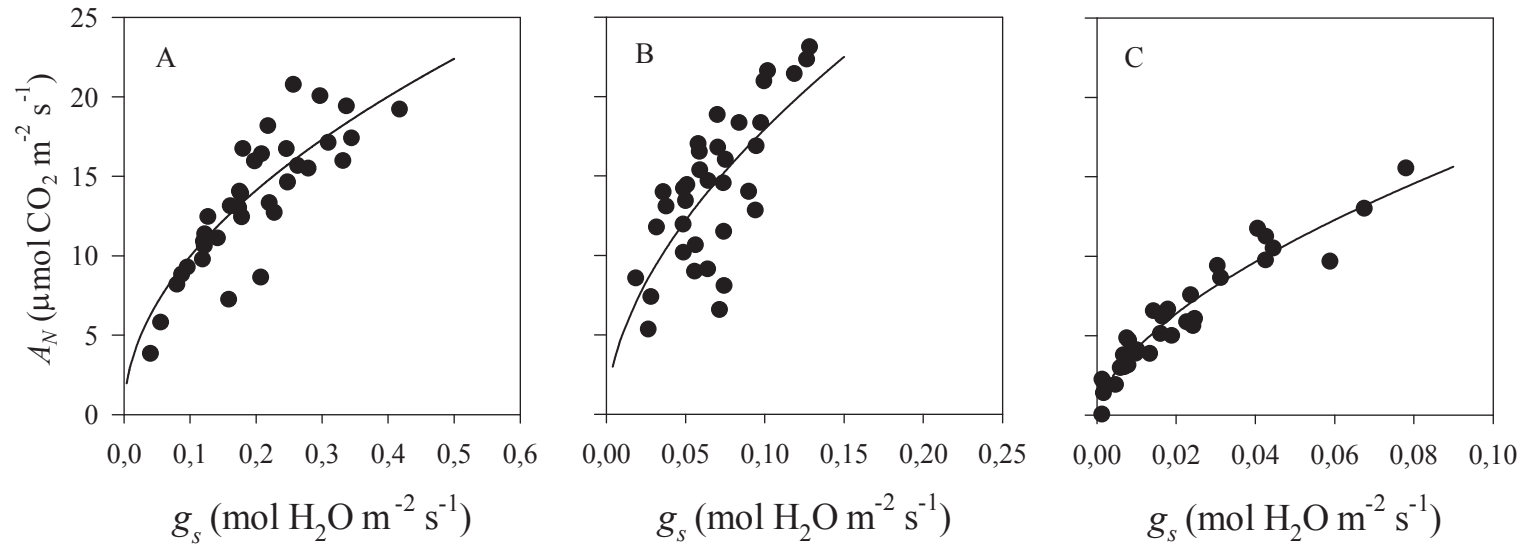

Figura 3. Relación entre la media de la tasa instantánea de fotosíntesis $\left(A_{N}, \mu \mathrm{mol} \mathrm{CO}_{2} \mathrm{~m}^{-2} \mathrm{~s}^{-1}\right)$ y la media de la conductancia estomática $\left(g_{s}\right.$, mol H $\left.\mathrm{O} \mathrm{m}^{-2} \mathrm{~s}^{-1}\right)$ de los 35 genotipos de Eucalyptus spp. medidos en el tratamiento de sequía en las instancias de muestreo equivalentes a A) -0,03 $\mathrm{MPa}, \mathrm{B})-1,5 \mathrm{MPa}$ y C) -2,5 $\mathrm{MPa}$ de potencial hídrico del sustrato.

Mean photosynthesis rate $\left(A_{N}, \mu \mathrm{mol} \mathrm{CO} \mathrm{m}^{-2} \mathrm{~s}^{-1}\right)$ and stomatal conductance $\left(g_{s}, \mathrm{~mol} \mathrm{H}_{2} \mathrm{O} \mathrm{m}^{-2} \mathrm{~s}^{-1}\right)$ relationship observed for all Eucalytpus spp. $(\mathrm{n}=35)$ genotypes at $-0.03 \mathrm{MPa}(\mathrm{M} 0),-1.5 \mathrm{MPa}(\mathrm{M} 1.5)$ and $-2.5 \mathrm{MPa}(\mathrm{M} 2.5)$ substrate water potential treatments. 

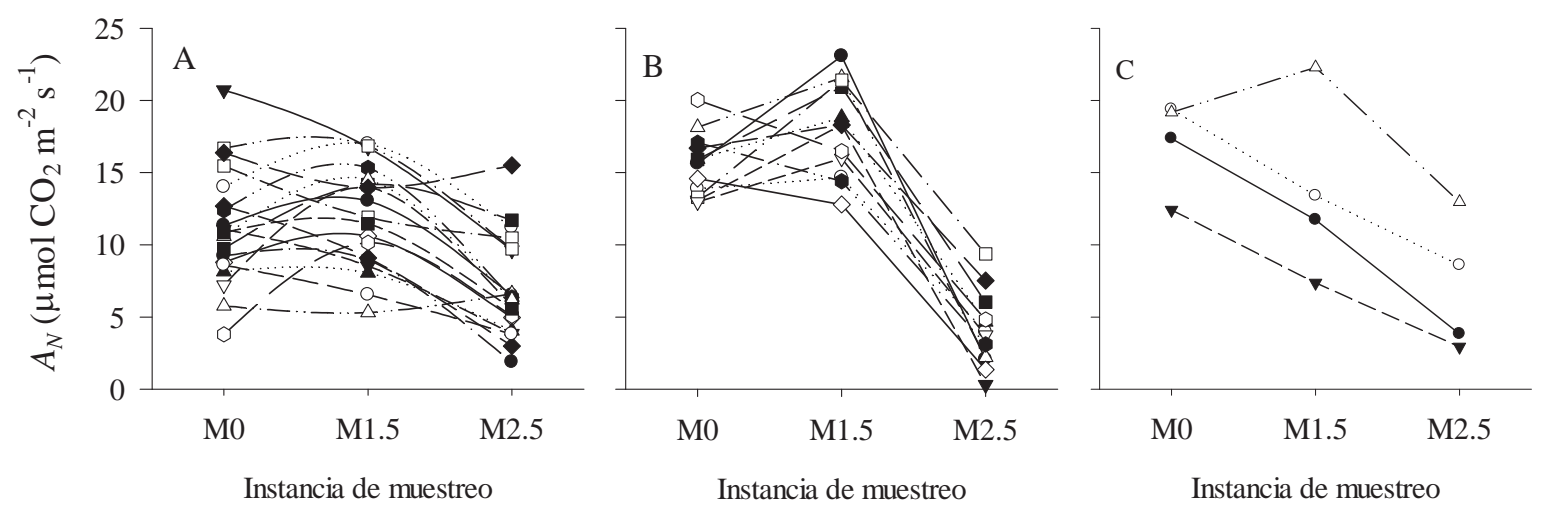

Figura 4. Media de la fotosíntesis instantánea máxima $\left(A_{N}, \mu \mathrm{mol} \mathrm{CO} \mathrm{C}^{-2} \mathrm{~s}^{-1}\right)$ para grupos de genotipos que en el tratamiento de sequía presentaron valores constantes entre instancias de muestreo (A), presentaron disminuciones significativas entre la segunda (M1.5) y tercera (M2.5) instancia de muestreo (B) y presentaron disminuciones significativas entre la primera (M0) y la tercera instancia de muestreo (M2.5) (C).

Mean photosynthesis rate $\left(A_{N}, \mu \mathrm{mol} \mathrm{CO} \mathrm{Cm}^{-2} \mathrm{~s}^{-1}\right)$ for genotypes groups under drought conditions that showed $\left.\mathrm{A}\right)$ constant values among sampling stages, B) significant reductions between the second (M1.5) and third (M2.5) treatment stages and C) significant reductions from the first (M0) to the third sampling stage (M2.5).

ensayo $(P<0,05)$. Un grupo de genotipos (Eng3, Eng4, Eng6, Eg1, Eg4, Eg13, Eg15, Eg16, Eg18, Eg19, Eg21, Eg22) disminuyó su fotosíntesis entre las instancias de muestreo finales (M1.5 y M2.5) (figura 4B) y otro grupo (Ecg, Es, Eg2 y Eg3) lo hizo solamente entre el muestreo inicial (M0) y el final (M2.5) (figura 4C).

Eficiencia instantánea en el uso de agua (EUAi). Diferencias significativas en eficiencia instantánea en el uso de agua se observaron entre los tratamientos $(P<0,05)$ en cada instancia de medición. Se observó un incremento progresivo en los valores promedio de $E U A_{i}$ desde 2,98 \pm 0,10 $\mu \mathrm{mol} \mathrm{CO} \mathrm{Cmol}^{-1} \mathrm{H}_{2} \mathrm{O}$ en la instancia de muestreo inicial (M0) hasta 13,62 $\pm 0,71 \mu \mathrm{mol} \mathrm{CO} \mathrm{mmol}^{-1} \mathrm{H}_{2} \mathrm{O}$ al final del ensayo (M2.5) (figura 5). Al contrario del comportamiento observado en la mayoría de los genotipos, al llegar a la segunda instancia de muestreo (M1.5) los genotipos Eg5, Eg6, Eg8, Eg11, Eg14, Eg20, Eg22, Eb no mostraron un aumento significativo en eficiencia instantánea en el uso de agua respecto a la primera medición. Hacia el final del ensayo los genotipos Eg6 y Eg14 mantuvieron el mismo comportamiento mencionado anteriormente, manteniendo valores promedio de $E U A_{i}$ similares durante todo el ensayo $(P>0,05)$. A diferencia de la primera instancia de muestreo (M0), en los muestreos consecuentes se observaron diferencias significativas entre los genotipos, siendo en el muestreo final (M2.5) donde se observó mayor dispersión de los datos (figura 5). No se observó una tendencia clara hacia cuál de las variables instantáneas de intercambio gaseoso fue causal de los cambios observados en $E U A_{i}$, obteniéndose una correlación negativa con todas ellas $(P<0,05)$. Los valores más altos observados en M2.5 se observaron en los genotipos Eg1, Eg16, Eg22 y Eng6, los cuales presentaron los valores más bajos de fotosíntesis, conductancia estomática y transpiración instantánea hacia el final del ensayo.

Supervivencia y relación con variables de intercambio gaseoso. El rango de supervivencia varió entre 0 y 70 \%, siendo los genotipos Eg1, Eg2, Eg3, Eg4, Eg14 y Eg22 los que

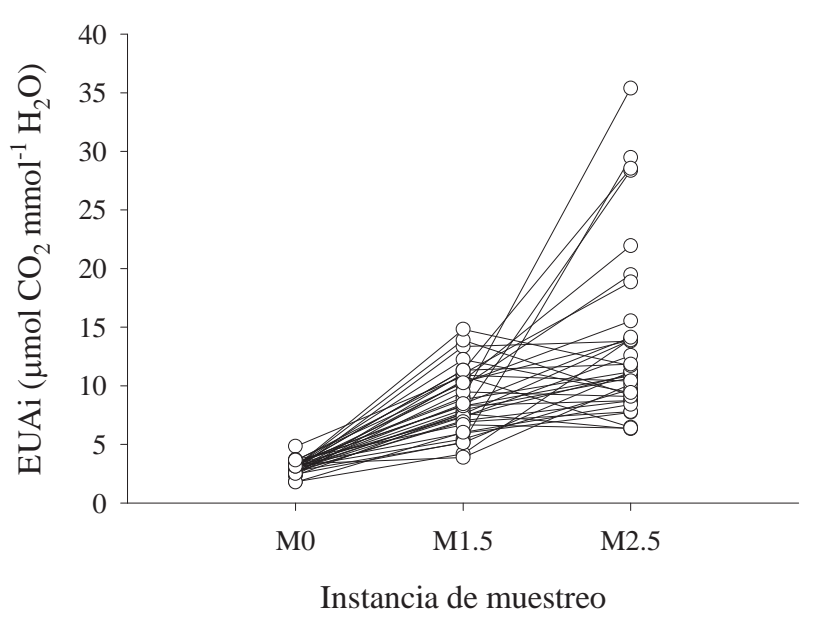

Figura 5. Media de la eficiencia instantánea en el uso de agua $\left(E U A_{i}, \mu m o l C_{2}\right.$ mmol $\left.^{-1} \mathrm{H}_{2} \mathrm{O}\right)$ medida a los 35 genotipos de $E$ ucalyptus spp. del tratamiento de sequía en las instancias de muestreo equivalentes a $-0,03 \mathrm{MPa}(\mathrm{M} 0),-1,5 \mathrm{MPa}(\mathrm{M} 1.5)$ y $-2,5 \mathrm{MPa}$ (M2.5) de potencial hídrico del sustrato.

Mean instantaneous water use efficiency $\left(E U A_{i}, \mu \mathrm{mol} \mathrm{CO}_{2}\right.$ $\left.\mathrm{mmol}^{-1} \mathrm{H}_{2} \mathrm{O}\right)$ observed for all Eucalytpus spp. $(\mathrm{n}=35)$ genotypes at -0.03 MPa (M0), -1.5 MPa (M1.5) and -2.5 MPa (M2.5) substrate water potential treatments. 
presentaron tasa de supervivencia de $0 \%$ y los genotipos Eg5, Eg7, Eg12 y Eg20 los que presentaron los valores más altos (60-70 \%). Se observó una correlación negativa entre el porcentaje de supervivencia y las variables instantáneas de intercambio gaseoso medidas en la primera instancia de muestreo $(P<0,05)$, excepto para eficiencia instantánea en el uso de agua $(P=0,23)$ (cuadro 1). Además, en la segunda instancia de muestreo, la fotosíntesis instantánea y la eficiencia instantánea en el uso de agua mostraron correlación negativa con la supervivencia $(P<0,05)$ (cuadro 1$)$. Una correlación positiva fue observada con el potencial hídrico foliar en las instancias de muestreo de estrés hídrico $(P<$ $0,05)$ (cuadro 1). Los genotipos con las más altas tasas de supervivencia presentaron un comportamiento similar entre instancias de muestreo en sus tasas fotosintéticas, disminución de su conductancia estomática y transpiración instantánea, y un aumento significativo de la eficiencia instantánea en el uso de agua hacia el final del ensayo. Además, aquellos genotipos que mantuvieron su conductancia estomática y transpiración bajas durante todo el ensayo presentaron tasas de supervivencia menores al $30 \%$. Por otra parte, todos los genotipos que disminuyeron significativamente la tasa de fotosíntesis entre instancias de muestreo presentaron supervivencias menores al $20 \%$, excepto, el genotipo Eng6 que registró una supervivencia de $45 \%$. Del mismo modo, aquellos genotipos que sufrieron la disminución más drástica de potencial hídrico foliar, hacia el final del ensayo, mostraron las más bajas tasas de supervivencia.

\section{DISCUSIÓN Y CONCLUSIONES}

Las respuestas en los parámetros de intercambio gaseoso de los genotipos bajo condiciones de déficit hídrico moderado (mayor a -1,5 $\mathrm{MPa}$ ) fueron más variables que para condiciones de mayor déficit hídrico (-2,5 MPa). Aquellos genotipos que no disminuyeron significativamente su potencial hídrico foliar en la primera etapa del ensayo, serían capaces de prolongar el estrés moderado o posponer el estrés severo para mantener mejores tasas de fotosíntesis y así reducir los efectos negativos causados por el déficit hídrico. Esta estrategia les permitiría resistir mejor las condiciones de déficit hídrico una vez establecidos en condiciones de campo, lo que coincide con lo observado en la relación entre el potencial hídrico foliar y la supervivencia en este estudio (cuadro 1).

En la mayoría de los genotipos, las variables de intercambio gaseoso (conductancia estomática, transpiración instantánea y tasa neta de fotosíntesis) presentaron reducciones significativas al aumentar el déficit hídrico, siendo la respuesta en conductancia estomática y transpiración más rápida que en fotosíntesis. Esto se ve reflejado en que las primeras solo alcanzan sus valores máximos cuando hay máxima disponibilidad de agua en el sustrato (figura 2), en cambio, la fotosíntesis se mantuvo constante entre las primeras instancias de muestreo en gran parte de los genotipos, disminuyendo significativamente solo al alcanzar el déficit hídrico más severo (figura 4). La relación observada entre conductancia estomática y fotosíntesis instantánea (figura 3) ha sido ampliamente reportada (Medrano et al. 2002, Flexas et al. 2010, 2013), estableciéndose que los máximos valores en las tasas fotosintéticas se alcanzan cuando la conductancia estomática es al menos la mitad que su máximo valor, es decir, existe un rango de conductancia estomática sobre el cual la fotosíntesis no aumenta significativamente. Flexas et al. (2013) señalan que cuando las tasas fotosintéticas son más dependientes de la conductancia del mesófílo su variación debido a cambios en la conductancia estomática es despreciable, como podría ser el caso de los genotipos encontrados en este estudio (figura 4A).

Aquellos genotipos con valores bajos de conductancia estomática en un rango más amplio de potencial hídrico foliar impiden el colapso del sistema hidráulico de la planta y su consecuente mortalidad (McDowell et al. 2008) al evitar la pérdida excesiva de agua hacia el ambiente y la cavitación del xilema. Esta estrategia explicaría los altos porcentajes de supervivencia medidos en genotipos cuya

Cuadro 1. Coeficientes de correlación $(\mathrm{n}=35)$ para el porcentaje de supervivencia y las variables instantáneas de intercambio gaseoso y potencial hídrico foliar de prealba evaluadas en las instancias de muestreo equivalentes a $-0,03 \mathrm{MPa}(\mathrm{M} 0),-1,5 \mathrm{MPa}(\mathrm{M} 1.5)$ y $-2,5$ MPa (M2.5) de potencial hídrico del sustrato.

Survival, gas exchange and predawn water potential correlation coefficients $(n=35)$ evaluated at $-0.03 \mathrm{MPa}(\mathrm{M} 0),-1.5 \mathrm{MPa}(\mathrm{M} 1.5)$ and -2.5 MPa (M2.5) substrate water potentials at each sampling stage.

\begin{tabular}{llcc}
\hline \multicolumn{1}{c}{ Variable } & M0 & M1.5 & M2.5 \\
\hline Fotosíntesis instantánea $\left(A_{N}\right)$ & $-0,5613 *$ & $-0,5258 *$ & $-0,0628 \mathrm{~ns}$ \\
Conductancia estomática $\left(g_{s}\right)$ & $-0,3993 *$ & $-0,1283 \mathrm{~ns}$ & $-0,0627 \mathrm{~ns}$ \\
Transpiración instantánea $(E)$ & $-0,4717 *$ & $-0,0875 \mathrm{~ns}$ & $-0,0283 \mathrm{~ns}$ \\
Eficiencia instantánea en el uso de agua $(E U A i)$ & $-0,2051 \mathrm{~ns}$ & $-0,4072 *$ & $-0,2819 \mathrm{~ns}$ \\
Potencial hídrico foliar de prealba $\left(\psi_{\text {prealba }}\right)$ & $0,1735 \mathrm{~ns}$ & $0,5814 *$ & $0,4154 *$ \\
\hline
\end{tabular}

* $P<0,05$; ns: $P>0,05$. 
conductancia estomática disminuyó durante el experimento y se mantuvo en valores cercanos a cero durante el déficit hídrico, siendo los genotipos que presentaron bajas tasas de conductancia estomática y transpiración aún en condiciones no limitantes de disponibilidad hídrica $(\mathrm{Eb}$, En1, Eg10, Eg14 y Eg24) más sensibles al estrés hídrico. Según lo observado en plantas de E. globulus sometidas a estrés por competencia con malezas por Garau et al. (2008), la disminución de la conductancia estomática en estrés hídrico moderado, como medio que permite posponer el estrés hídrico, sería una estrategia potencial para aumentar la supervivencia. Sin embargo, Eksteen et al. (2013) reportaron para la misma especie que la rápida disminución de esta variable ante el estrés hídrico también se relaciona con bajas tasas de crecimiento, lo que podría generar que en la primera etapa del establecimiento de plantaciones aumente la competencia con malezas por luz, agua y nutrientes incrementando el estrés hídrico y disminuyendo la disponibilidad de otros recursos.

La disminución de la conductancia estomática ha sido reportada como una de las principales limitantes de la fotosíntesis (Pita et al. 2005), lo que consecuentemente disminuiría las tasas de crecimiento. La disminución de las tasas de fotosíntesis se generó cuando la conductancia estomática alcanzó valores menores de 0,10 moles de $\mathrm{H}_{2} \mathrm{O} \mathrm{m}^{-2}$ $\mathrm{s}^{-1}$, lo cual concuerda con los datos recopilados por Flexas et al. (2006), quienes señalan que bajo este rango de conductancia estomática el estrés hídrico aumenta las limitaciones bioquímicas, además de disminuir la concentración de $\mathrm{CO}_{2}$ interno $\left(C_{i}\right)$ y por ende se produce la inhibición de la fotosíntesis. Los genotipos que no presentaron disminuciones en las tasas fotosintéticas (figura 4A) fueron aquellos que al menos en una instancia de muestreo no disminuyeron su conductancia estomática con respecto a la medición anterior, lo cual sugiere una estrategia de tipo menos conservadora del agua comparada a otros genotipos frente a señales de estrés hídrico temprano. Según lo observado por Flexas et al. (2006), al posponer el cierre estomático se retrasa la acumulación de antioxidantes, generándose estrés oxidativo que sería la causa principal de las respuestas metabólicas asociadas al estrés hídrico y que generan la inhibición de las tasas fotosintéticas y la consecuente disminución del crecimiento.

Las diferencias observadas en la eficiencia instantánea en el uso de agua están asociadas a variaciones en fotosíntesis instantánea, conductancia estomática y transpiración instantánea. De acuerdo a Flexas et al. (2013) el aumento en la eficiencia en el uso de agua está dado tanto por disminuciones en la conductancia estomática y reducciones en las tasas de fotosíntesis. Acorde a lo esperado, en esta investigación la mayor parte de los genotipos aumentaron la eficiencia instantánea en el uso de agua en las distintas condiciones de sequía con respecto al valor medido sin déficit hídrico (figura 5). Se encontró que los aumentos en la tasa fotosintética registrados en la segunda instancia de muestreo (M1.5) no generaron los valores más altos de eficiencia instantánea en el uso de agua, sino que estos se registraron en la instancia de estrés hídrico más severo (M2.5) donde la disminución en conductancia estomática y transpiración fue mucho mayor que la disminución en la fotosíntesis instantánea. Los resultados de este estudio muestran que la tasa fotosintética no disminuyó significativamente en la mayoría de los genotipos (figura 4A), concordando con lo observado por Navarrete et al. (2013), quienes en genotipos de E. globulus e híbridos E. globulus $x$ E. nitens reportaron que la mantención de altas tasas de fotosíntesis bajo condiciones de déficit hídrico, junto con un alto control estomático de la pérdida de agua generó el aumento de la eficiencia instantánea del uso de agua.

La relación negativa observada entre el porcentaje de supervivencia y las variables de intercambio gaseoso (cuadro 1), permitiría inferir que aquellos genotipos que mantienen tasas bajas de intercambio gaseoso durante el estrés hídrico moderado, o que presentan la disminución paulatina de variables como la conductancia estomática, serían capaces de disminuir la pérdida de agua y lograr mayor éxito en el establecimiento (Garau et al. 2008). Además, la relación negativa observada entre la fotosíntesis instantánea y la supervivencia en condiciones de alta disponibilidad de agua y estrés hídrico moderado (potencial hídrico de sustrato de -1,5 MPa), sugiere que la evaluación de esta variable permitiría la selección de genotipos con mayor posibilidad de éxito en el establecimiento en sitios con disponibilidad hídrica restringida. Sin embargo, los genotipos con las mayores tasas de supervivencia fueron aquellos que disminuyeron significativamente su conductancia estomática y mantuvieron su fotosíntesis instantánea constante a lo largo del ensayo. Además, todos los genotipos que disminuyeron su fotosíntesis instantánea como respuesta al estrés hídrico, mostraron tasas de supervivencia menores al $20 \%$, exceptuando el genotipo Eng6 que mostró altos valores en las variables de intercambio gaseoso y eficiencia instantánea en el uso de agua en las primeras dos instancias de muestreo. Dado el comportamiento fisiológico de este genotipo se sugiere que este podría presentar mejor crecimiento en condiciones de estrés hídrico moderado pero con baja probabilidad de supervivencia frente a condiciones de estrés severo o prolongado.

Los resultados de este estudio permiten aceptar la hipótesis respecto a que la evaluación de la tasa de fotosíntesis en condiciones de estrés hídrico moderado a nulo permite identificar materiales genéticos que potencialmente presentarían mayor supervivencia en sitios con disponibilidad hídrica limitante en etapas tempranas de desarrollo. Esta variable podría ser considerada para la selección de genotipos que presenten mayores tasas de supervivencia en sitios con baja disponibilidad hídrica al establecimiento.

\section{AGRADECIMIENTOS}

Esta investigación fue realizada como parte del proyecto FONDEF D11I1161 con aportes de Forestal Mininco 
S.A., Forestal Arauco S.A., Bioforest S.A., el Laboratorio de Suelos, Nutrición y Productividad Forestal Sustentable de la Facultad de Ciencias Forestales de la Universidad de Concepción y la Cooperativa de Productividad Forestal.

\section{REFERENCIAS}

Chaves MM, J Flexas, C Pinheiro. 2009. Photosynthesis under drought and salt stress: regulation mechanisms from whole plant to cell. Annals of Botany 103: 551-560.

Chaves MM, J Maroco, JS Pereira. 2003. Understanding plant responses to drought: from genes to the whole plant. Functional Plant Biology 30: 239-264.

Chaves MM, JS Pereira, J Maroco, ML Rodrigues, CPP Ricardo, ML Osorio, I Carvalho, T Faria, C Pinheiro. 2002. How plants cope with water stress in the field. Photosynthesis and growth. Annals of Botany 89:907-916.

Coopman R, J Jara, L Bravo, K Sáez, G Mella, R Escobar. 2008. Changes in morpho-physiological attributes of Eucalyptus globulus plants in response to different drought hardening treatments. Electronic Journal of Biotechnology 11(2).

Costa e Silva F, A Shvaleva, JP Maroco, MH Almeida, MM Chaves, JS Pereira. 2004. Responses to water stress in two Eucalyptus globulus clones differing in drought tolerance. Tree Physiology 24: 1165-1172.

Dye PJ. 2000. Water use efficiency in South African Eucalyptus plantations: A review. The Southern African Forestry Journal 189: 17-26.

Eksteen AB, V Grzeskowiak, NB Jones, NW Pammenter. 2013. Stomatal characteristics of Eucalyptus grandis clonal hybrids in response to water stress. Southern Forests: a Journal of Forest Science 75: 105-111.

Flexas J, M Barón, J Bota, J-M Ducruet, A Gallé, J Galmés, M Jiménez, A Pou, M Ribas-Carbó, C Sajnani, M Tomàs, $H$ Medrano. 2009. Photosynthesis limitations during water stress acclimation and recovery in the drought-adapted Vitis hybrid Richter-110 ( $V$. berlandieri $\times V$. rupestris). Journal of Experimental Botany 60: 2361-2377.

Flexas J, J Bota, F Loreto, G Cornic, TD Sharkey. 2004. Diffusive and metabolic limitations to photosynthesis under drought and salinity in C3 plants. Plant Biology 6: 269-279.

Flexas J, Ü Niinemets, A Gallé, MM Barbour, M Centritto, A Diaz-Espejo, C Douthe, J Galmés, M Ribas-Carbo, PL Rodriguez, F Rosselló, R Soolanayakanahally, M Tomas, IJ Wright, GD Farquhar, H Medrano. 2013. Diffusional conductances to $\mathrm{CO}_{2}$ as a target for increasing photosynthesis and photosynthetic water-use efficiency. Photosynthesis Research 117: 45-59.
Galmés J, H Medrano, J Flexas. 2007. Photosynthetic limitations in response to water stress and recovery in Mediterranean plants with different growth forms. New Phytologist 175: 81-93.

Garau AM, JH Lemcoff, CM Ghersa, CL Beadle. 2008. Water stress tolerance in Eucalyptus globulus Labill. subsp. maidenii (F. Muell.) saplings induced by water restrictions imposed by weeds. Forest Ecology and Management 255: 2811-2819.

Hubbard RM, J Stape, MG Ryan, AC Almeida, J Rojas. 2010. Effects of irrigation on water use and water use efficiency in two fast growing Eucalyptus plantations. Forest Ecology and Management 259: 1714-1721.

INFOR (Instituto Forestal, CL). 2015. Anuario Forestal 2015. Santiago, Chile. INFOR. 174 p. (Boletín Estadístico $\mathrm{N}^{\circ}$ 150).

Li C. 2000. Population differences in water-use efficiency of Eucalyptus microtheca seedlings under different watering regimes. Physiologia Plantarum 108: 134-139.

Li C, K Wang. 2003. Differences in drought responses of three contrasting Eucalyptus microtheca F. Muell. populations. Forest Ecology and Management 179: 377-385.

McDowell N. W T Pockman, CD Allen, DD Breshears, N Cobb, T Kolb, J PLaut, J Sperry, A West, DG Williams, EA Yepez. 2008. Mechanisms of plant survival and mortality during drought: why do some plants survive while others succumb to drought? New Phytologist 178: 719-739.

Medrano H, J Bota, A Abadía, B Sampol, JM Escalona, J Flexas. 2002. Effects of drought on light-energy dissipation mechanisms in high-light-acclimated, field-grown grapevines. Functional Plant Biology 29: 1197-1207.

Medrano H., J. Bota, J. Cifre, J. Flexas, M. Ribas-Carbó y J. Gulías. 2007. Eficiencia en el uso de agua por las plantas. Investigaciones Geográficas 43: 63-84.

Navarrete-Campos D, LA Bravo, RA Rubilar, V Emhart, R Sanhueza. 2012. Drought effects on water use efficiency, freezing tolerance and survival of Eucalyptus globulus and Eucalyptus globulus $\times$ nitens cuttings. New Forests 44: 119-134.

Pardos JA. 2007. Perspectiva fisiológica en la producción y mejora del eucalipto (con énfasis en Eucalyptus globulus Labill). Boletín del CIDEU 3: 7-55.

Pita P, I Cañas, F Soria, F Ruiz, G Toval. 2005. Use of physiological traits in tree breeding for improved yield in droughtprone environments. The case of Eucalyptus globulus. Investigación Agraria: Sistemas y Recursos Forestales 14(3): 383-393.

Wikberg J, E Ögren. 2003. Interrelationships between water use and growth traits in biomass-producing willows. Trees 18: 70-76. 
